\title{
Calculation of Vibrational Frequencies of Sulfur Dioxide by Lie Algebraic Framework
}

\author{
M.R. BAlla ${ }^{a}$, S. Venigalla ${ }^{b}$ And V. JALiparThi ${ }^{c, *}$ \\ ${ }^{a}$ Department of Physics, School of Science, GITAM Deemed-to-be-University, \\ Rudraram, 502329, Hyderabad, India \\ ${ }^{b}$ Department of Chemistry, A.G.\& S.G. Siddhartha Degree College of Arts $\&$ Science, \\ Vuyyuru, 521165, Vijayawada, India \\ ${ }^{c}$ Department of Mathematics, School of Science, GITAM Deemed-to-be-University, \\ Rudraram, 502329, Hyderabad, India
}

Received: 05.05.2021 \& Accepted: 20.07.2021

Doi: 10.12693/APhysPolA.140.138

*e-mail: vijayjaliparthi@gmail.com

\begin{abstract}
In this paper, we have demonstrated the application of the $\mathrm{U}(2)$ Lie algebraic method to predict the vibrational frequencies of sulfur dioxide $\left(\mathrm{SO}_{2}\right)$. A Hamiltonian that preserves the $C_{2 \nu}$ point group symmetry of the molecule is devised using three interacting Morse oscillators. Root mean square deviation of the calculated vibrational frequencies is found to be $1.054 \mathrm{~cm}^{-1}$ with reference to their experimental values. This asserts that the $\mathrm{U}(2)$ Lie algebraic method is successful in calculating the fundamental vibrational frequencies, and their higher overtones near to the spectroscopic level of accuracy.
\end{abstract}

topics: vibrational frequencies, sulfur dioxide, $\mathrm{U}(2)$ Lie algebraic method

\section{Introduction}

Theoretical calculations of vibrational frequencies of polyatomic molecules have been one of the interesting research areas for scientists on account of the development of innovative spectroscopic techniques. The investigation of vibrational spectra of molecules has been improved in recent years both theoretically and experimentally. Two approaches have been predominantly used so far in the study of experimental spectra: the well-known Dunhamlike expansion of energy levels in terms of rotationvibration quantum numbers and the solution of the Schrödinger equation with potential functions.

In this work, we applied the Lie algebraic method to study the vibrational frequencies of sulfur dioxide. This method reformulates the Hamiltonian operator in terms of elements of the Lie algebra and provides the same physical information as that of the Dunham and potential approaches $[1,2]$. The advantage of the proposed method, as compared to that of the Dunham or potential approach, is that usually fewer parameters are required to get the same level of accuracy, in contrast to their comparators $[3,4]$. The Lie algebraic method makes it possible to predict the vibrational frequencies much more accurately and possibly at a much lesser computational cost as compared to other theoretical approaches. The lesser computational cost of the Lie algebraic framework is evident from the lesser demand of computational time for performing algebraic manipulations, rather than integration and differentiation of the potential function as in other approaches $[1,2]$.

\section{The $U(2)$ Lie algebraic method}

Sulfur dioxide $\left(\mathrm{SO}_{2}\right)$ is a bent triatomic molecule with the equilibrium structure belonging to the $C_{2 \nu}$ point group symmetry. The molecule is non-linear with 3 vibrational degrees of freedom. Each of the vibrating bonds in $\mathrm{SO}_{2}$ molecule is effectively described by a one-dimensional Morse oscillator and is assigned with a corresponding $\mathrm{U}(2)$ Lie algebra, as per the schematic shown in Fig. 1.

The two possible chains of dynamical symmetry groups in $\mathrm{SO}_{2}$ molecule, corresponding to the local and normal couplings in stretching vibrations, are given by

$$
\begin{aligned}
& U_{1}(2) \otimes U_{2}(2) \supset O_{1}(2) \otimes O_{2}(2) \rightarrow \underset{\text { local }}{\text { coupling }} \\
& U_{1}(2) \otimes U_{2}(2) \supset U_{12}(2) \supset O_{12}(2) \rightarrow \text { normal } \\
& \text { coupling }
\end{aligned}
$$

The interaction results in three normal mode vibrations $\nu_{1}, \nu_{2}$, and $\nu_{3}$, correspond to the symmetry species $\mathrm{A}_{1}$ (symmetric stretch), $\mathrm{B}_{1}$ (asymmetric stretch), and $A_{1}$ (bend). 


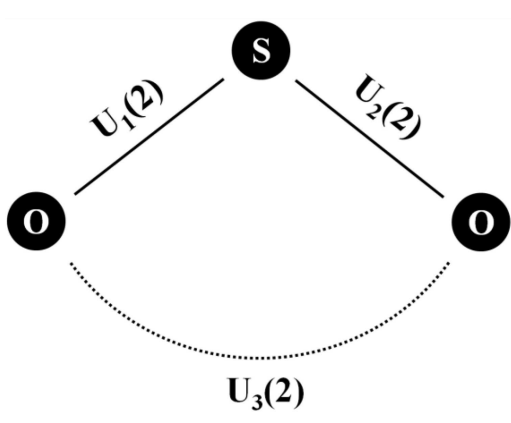

Fig. 1. Assignment of $\mathrm{U}(2)$ Lie algebras for bonds in $\mathrm{SO}_{2}$ molecule.

The local vibrational basis is constructed as

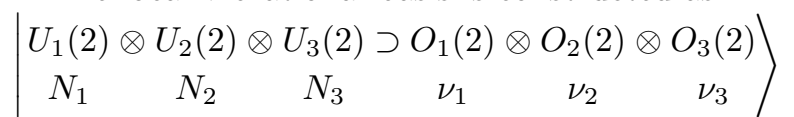

keeping the total vibrational quantum number $V=\sum_{i=a, b, c} \nu_{i}$ always a conserved quantity.

The Hamiltonian operator that describes the vibrational spectra of the two interacting single bonds $(\mathrm{S}-\mathrm{O})$ in $\mathrm{SO}_{2}$ molecule are expressed mathematically as $[5,6]$ :

$$
H=E_{0}+\sum_{i=1}^{n} A_{i} C_{i}+\sum_{i<j}^{n} A_{i j} C_{i j}+\sum_{i<j}^{n} \lambda_{i j} M_{i j} .
$$

Here, $E_{0}$ is the electronic ground state energy of the bond $\mathrm{S}-\mathrm{O}$, which will be taken as the zero reference for all the vibrational excitations. The term $\sum_{i=1}^{n} A_{i} C_{i}$ corresponds to the independent local oscillators expressed using the invariant Casimir operators $C_{i}$. The term $\sum_{i<j}^{n} A_{i j} C_{i j}$ is accounted for the cross-anharmonicities between pairs of distinct local oscillators in terms of the coupled Casimir operators $C_{i j}$. The term $\sum_{i<j}^{n} \lambda_{i j} M_{i j}$ expresses the anharmonic, non-diagonal interactions involving the pairs of local oscillators in terms of the coupled Majorana operators $M_{i j}$. The eigenvalues of the Hamiltonian can be evaluated and give a description of $n$ coupled anharmonic vibrations. The couplings in the Hamiltonian are only first order, in the sense that the Majorana operators $M_{i j}$ annihilate one quantum of vibration in bond $j$, and create one in bond $i$ (or vice versa).

Here, $i$ varies from 1 to 2 for the two $(\mathrm{S}-\mathrm{O})$ stretching bonds. The algebraic parameters $\left\{A_{i}, A_{i j}, \lambda_{i j}\right\}$ are calculated from the spectroscopic data of $\mathrm{S}-\mathrm{O}$ single bond. The invariant operator $C_{i}$ of the uncoupled bond with eigenvalues $-4\left(N_{i} \nu_{i}-\nu_{i}^{2}\right)$ and the operator $C_{i j}$ for coupled bonds are diagonal with matrix elements

$$
\begin{aligned}
& \left\langle N_{i}, \nu_{i} ; N_{j}, \nu_{j}\left|C_{i j}\right| N_{i}, \nu_{i} ; N_{j}, \nu_{j}\right\rangle= \\
& 4\left(\left(\nu_{i}+\nu_{j}\right)^{2}-\left(\nu_{i}+\nu_{j}\right)\left(N_{i}+N_{j}\right)\right),
\end{aligned}
$$

while the Majorana operator $M_{i j}$ has both diagonal and non-diagonal matrix elements

$$
\begin{aligned}
& \left\langle N_{i}, \nu_{i} ; N_{j}, \nu_{j}\left|M_{i j}\right| N_{i}, \nu_{i} ; N_{j}, \nu_{j}\right\rangle= \\
& \left(N_{i} \nu_{j}+N_{j} \nu_{i}-2 \nu_{i} \nu_{j}\right), \\
& \left\langle N_{i}, \nu_{i}+1 ; N_{j}, \nu_{j}-1\left|M_{i j}\right| N_{i}, \nu_{i} ; N_{j}, \nu_{j}\right\rangle= \\
& -\sqrt{\nu_{j}\left(\nu_{i}+1\right)\left(N_{i}-\nu_{i}\right)\left(N_{j}-\nu_{j}+1\right)}, \\
& \left\langle N_{i}, \nu_{i}-1 ; N_{j}, \nu_{j}+1\left|M_{i j}\right| N_{i}, \nu_{i} ; N_{j}, \nu_{j}\right\rangle= \\
& -\sqrt{\nu_{i}\left(\nu_{j}+1\right)\left(N_{j}-\nu_{j}\right)\left(N_{i}-\nu_{i}+1\right)},
\end{aligned}
$$

where $\nu_{i}(i=1,2, \ldots)$ are the vibrational quantum numbers. The vibron number $N_{i}(i=1,2)$ for (S-O) stretching bonds of the molecule are calculated by the following relation [7]:

$$
N_{i}=\frac{\omega_{e}}{\omega_{e} x_{e}}-1 \text {. }
$$

Here, $U_{i}(2)$ are the spectroscopic constants [8] and $1 \leqslant i \leqslant 3$. The initial guess value for the parameter $A_{i}(i=1,2)$ is obtained by using the energy equation for the single-oscillator fundamental mode. It is given as [9-11]:

$$
E(\nu=1)=-4 A_{i}\left(N_{i}-1\right) .
$$

Initial guesses for $A_{i j}$ are taken as zero. The parameter $\lambda_{i j}$ is determined from the relation $[1,2]$ :

$$
\lambda_{i j} \simeq \frac{\left|E_{i}-E_{j}\right|}{2 N} .
$$

To get accurate results, a numerical fitting procedure is essential to obtain the parameters $A_{i}, \lambda_{i j}$ (when $i, j=1,2$, and $i \neq j$ ) starting from values as given by (8) and (9).

\section{Results}

Our calculations based on the constructed Lie algebraic Hamiltonian, followed by the linear regression analysis (fitting procedure) resulted in the optimized algebraic parameters, which are given in Table I.

The calculated vibrational frequencies in fundamental, overtone and their combinations are presented in Table II.

\section{TABLE I}

Optimised fitting parameters. Note: $N_{1}, N_{2}$, and $N_{3}$ are dimensionless numbers.

\begin{tabular}{c|c|l}
\hline \hline $\begin{array}{c}\text { U(2) Lie algebraic } \\
\text { model parameters }\end{array}$ & Optimised value & $\begin{array}{l}\text { Vibrational } \\
\text { mode }\end{array}$ \\
\hline$N_{1}, N_{2}$ & 178 & stretching \\
$N_{3}$ & 88 & bending \\
$A_{1}, A_{2}$ & $-2.2799 \mathrm{~cm}^{-1}$ & stretching \\
$A_{3}$ & $-2.2297 \mathrm{~cm}^{-1}$ & bending \\
$A_{12}, A_{21}$ & $0.3262 \mathrm{~cm}^{-1}$ & stretching \\
$A_{13}, A_{23}$ & $0.3699 \mathrm{~cm}^{-1}$ & bending \\
$\lambda_{12}, \lambda_{21}$ & $0.5905 \mathrm{~cm}^{-1}$ & stretching \\
$\lambda_{13}, \lambda_{23}$ & $2.9425 \mathrm{~cm}^{-1}$ & bending \\
\end{tabular}


TABLE II

Vibrational frequencies of sulfur dioxide.

\begin{tabular}{|c|c|c|}
\hline \multirow{2}{*}{$\begin{array}{c}\text { Vibrational } \\
\text { mode }\end{array}$} & \multicolumn{2}{|c|}{ Vibrational frequencies $\left[\mathrm{cm}^{-1}\right]$} \\
\hline & Experiment $[12,13]$ & $U(2)$ Lie method \\
\hline$\left(\begin{array}{lll}1 & 0 & 0\end{array}\right)$ & 1151 & 1150.9652 \\
\hline$\left(\begin{array}{lll}0 & 0 & 1\end{array}\right)$ & 1361.2 & 1361.1832 \\
\hline$\left(\begin{array}{lll}0 & 1 & 0\end{array}\right)$ & 517 & 517.8944 \\
\hline$\left(\begin{array}{lll}2 & 0 & 0\end{array}\right)$ & 2295.9 & 2294.9032 \\
\hline$\left(\begin{array}{lll}0 & 0 & 2\end{array}\right)$ & 2714 & 2715.6130 \\
\hline$\left(\begin{array}{lll}0 & 2 & 0\end{array}\right)$ & 1034.9 & 1035.7744 \\
\hline$\left(\begin{array}{lll}3 & 0 & 0\end{array}\right)$ & 3435.4 & 3437.0171 \\
\hline$\left(\begin{array}{lll}0 & 0 & 3\end{array}\right)$ & - & 4056.5053 \\
\hline$\left(\begin{array}{lll}0 & 3 & 0\end{array}\right)$ & - & 1553.6544 \\
\hline$\left(\begin{array}{lll}4 & 0 & 0\end{array}\right)$ & - & 4558.1320 \\
\hline$\left(\begin{array}{lll}0 & 0 & 4\end{array}\right)$ & - & 5403.2261 \\
\hline$\left(\begin{array}{lll}0 & 4 & 0\end{array}\right)$ & - & 2071.5344 \\
\hline$\left(\begin{array}{lll}5 & 0 & 0\end{array}\right)$ & - & 5758.4300 \\
\hline$\left(\begin{array}{lll}0 & 0 & 5\end{array}\right)$ & - & 6845.2126 \\
\hline$\left(\begin{array}{lll}0 & 5 & 0\end{array}\right)$ & - & 2589.4144 \\
\hline$\left(\begin{array}{lll}6 & 0 & 0\end{array}\right)$ & - & 6815.3209 \\
\hline$\left(\begin{array}{lll}0 & 0 & 6\end{array}\right)$ & - & 8287.0553 \\
\hline$\left(\begin{array}{lll}0 & 6 & 0\end{array}\right)$ & - & 3107.2944 \\
\hline$\left(\begin{array}{lll}1 & 0 & 1\end{array}\right)$ & 2499.1 & 2514.758 \\
\hline$\left(\begin{array}{lll}2 & 0 & 1\end{array}\right)$ & - & 3658.696 \\
\hline$\left(\begin{array}{lll}1 & 0 & 2\end{array}\right)$ & 3837 & 3869.1878 \\
\hline$\left(\begin{array}{lll}3 & 0 & 1\end{array}\right)$ & - & 4800.8099 \\
\hline$\left(\begin{array}{lll}1 & 0 & 3\end{array}\right)$ & - & 5210.0801 \\
\hline$\left(\begin{array}{lll}4 & 0 & 1\end{array}\right)$ & - & 5921.9248 \\
\hline$\left(\begin{array}{lll}1 & 0 & 4\end{array}\right)$ & - & 6556.8009 \\
\hline$\left(\begin{array}{lll}5 & 0 & 1\end{array}\right)$ & - & 7122.2228 \\
\hline$\left(\begin{array}{lll}1 & 0 & 5\end{array}\right)$ & - & 7998.7874 \\
\hline$\left(\begin{array}{lll}2 & 0 & 2\end{array}\right)$ & - & 5013.1258 \\
\hline$\left(\begin{array}{lll}3 & 0 & 3\end{array}\right)$ & - & 7496.132 \\
\hline
\end{tabular}

\section{Conclusion}

Vibrational frequencies of sulfur dioxide are calculated using the $\mathrm{U}(2)$ Lie algebraic method, and the calculated frequencies are compared with the reported experimental data. The results obtained are found to be consistent with the experimental results, indicating that our approach can be applied to study the vibrational spectra of a variety of molecules. The obtained results confirm that the $\mathrm{U}(2)$ Lie algebraic framework has successfully reproduced the vibrational frequencies at the fundamental excitations near to the level of spectroscopic accuracy, as well as predicted their first five overtones.

\section{References}

[1] S. Oss, Adv. Chem. Phys. 93, 455 (1996).

[2] F. Iachello, R.D. Levine, Algebraic Theory of Molecules, Oxford University Press, Oxford 1995.

[3] N.K. Sarkar, Mol. Phys. 119, e1836409 (2021).

[4] S.R. Karumuri, K.G. Sravani, J. Vijayasekhar, L.S.S. Reddy, Acta Phys. Pol. A 122, 1111 (2012).

[5] M.R. Balla, V.S. Jaliparthi, Mol. Phys. 115, e1828634 (2021).

[6] F. Iachello, Chem. Phys. Lett. 78, 581 (1981).

[7] F. Iachello, R.D. Levine, J. Chem. Phys. 77, 3046 (1982).

[8] K.K. Irikura, J. Phys. Chem. Ref. Data 36, 389 (2007).

[9] F. Iachello, S. Oss, J. Mol. Spectrosc. 142, 85 (1990).

[10] F. Zhaochi, G. Xiong, Y. Qihua, Q. Xin, Li Can, Chin. Sci. Bull. 44, 1961 (1999).

[11] M.R. Balla, V.S. Jaliparthi, "Vibrational Hamiltonian of Naphthalene $\left(\mathrm{C}_{10} \mathrm{H}_{8}\right)$ Using Dynamical U(2) Lie Algebras", Polycycl. Aromat. Compd., (2021).

[12] W.S. Benedict, N. Gailar, E.K. Plyler, J. Chem. Phys. 21, 1301 (1953).

[13] W.S. Benedict, N. Gailar, E.K. Plyler, J. Chem. Phys. 24, 1139 (1956). 